\title{
THE FINITISTIC DIMENSION OF AN ARTIN ALGEBRA WITH RADICAL SQUARE ZERO
}

\author{
VINCENT GÉLINAS
}

\begin{abstract}
We investigate the inequality Findim $\Lambda^{o p} \leq$ dell $\Lambda$ between the finitistic dimension and the delooping level of an Artin algebra $\Lambda$, and whether equality holds in general. We prove that equality Findim $\Lambda^{o p}=\operatorname{dell} \Lambda$ always holds for Artin algebras with radical square zero.
\end{abstract}

\section{INTRODUCTION}

The little finitistic dimension findim $\Lambda$ and big finitistic dimension Findim $\Lambda$ are important homological invariants of Artin algebras $\Lambda$. However, understanding their values in terms of more basic invariants of $\Lambda$ remains difficult.

In the setting of commutative local Noetherian rings $R=(R, \mathfrak{m}, k)$, the analogous question has a satisfactory answer: the little finitistic dimension findim $R=\operatorname{depth} R$ is equal to the depth of $R$ by the Auslander-Buchsbaum formula, and the big finitistic dimension Findim $R=\operatorname{dim} R$ equals its Krull dimension by theorems of Bass [Bas62] and Gruson-Raynaud [GR71].

The depth of $R$ can be defined in terms of the Ext functor via $\operatorname{Ext}_{R}^{*}(k, R)$, and so admits a natural extension to the setting of Noetherian semiperfect rings $\Lambda$, and in particular to that of Artin algebras ([Gé20, Section 1]). In this situation, the Auslander-Buchsbaum equality is replaced by an inequality due to Jans [Jan61]

$$
\operatorname{depth} \Lambda \leq \operatorname{findim} \Lambda^{o p}
$$

between the (right) depth of $\Lambda$ and its (left) little finitistic dimension. However, equality fails to hold in general. In [Gé20], the author introduced a new invariant dell $\Lambda$ for Noetherian semiperfect rings $\Lambda$, taking value in $\mathbb{N} \cup\{\infty\}$, which refines Jans' inequality to

$$
\operatorname{depth} \Lambda \leq \operatorname{findim} \Lambda^{o p} \leq \operatorname{dell} \Lambda
$$

and gives rise for Artin algebras $\Lambda$ to finer inequalities

$$
\operatorname{depth} \Lambda \leq \text { findim } \Lambda^{o p} \leq \operatorname{Findim} \Lambda^{o p} \leq \operatorname{dell} \Lambda .
$$

This new invariant was shown to recover the depth for commutative local Noetherian rings $R$ in that depth $R=\operatorname{dell} R$, so that the above collapse to equalities in the commutative case. Similar results hold for Noetherian semiperfect rings $\Lambda$ satisfying certain cohomological conditions (see [Gé20, Thm. 2.12]). However, the above inequalities are strict in general. 
In this paper, we restrict ourselves to the setting of Artin algebras $\Lambda$. Of the three inequalities

$$
\operatorname{depth} \Lambda \leq \operatorname{findim} \Lambda^{o p} \leq \operatorname{Findim} \Lambda^{o p} \leq \operatorname{dell} \Lambda .
$$

the first two depth $\Lambda \leq$ findim $\Lambda^{o p}$ and findim $\Lambda^{o p} \leq$ Findim $\Lambda^{o p}$ are known to be strict in some examples; however, the last inequality

$$
\text { Findim } \Lambda^{o p} \leq \operatorname{dell} \Lambda
$$

is less understood. In [Gé20], it was shown to be a form of the "Cohen-Macaulay" property. The following curious result was observed:

Theorem ([Gé20, Thm. 4.1]). Let $\Lambda$ be a Noetherian semiperfect ring, and consider the inequality Findim $\Lambda^{o p} \leq \operatorname{dell} \Lambda$.

i) The inequality characterises Cohen-Macaulay rings amongst commutative local Noetherian rings, in which case we have equality.

ii) The inequality holds for all Gorenstein rings, in which case we have equality.

iii) The inequality holds for Artinian rings.

In the first two cases, the inequality is actually an equality: when $R=(R, \mathfrak{m}, k)$ is commutative local Noetherian the inequality boils down to $\operatorname{dim} R \leq \operatorname{depth} R$, which immediately forces $\operatorname{dim} R=\operatorname{depth} R$. For noncommutative Gorenstein rings $\Lambda$, the equality Findim $\Lambda^{o p}=\operatorname{dell} \Lambda$ is a consequence of powerful results of AngeleriHügel-Herbera-Trlifaj [AHHT06]. This leaves the case of Artinian rings open, and leads to the natural question:

Question ([Gé20, Q.4.2]). Does Findim $\Lambda^{o p}=$ dell $\Lambda$ hold for all Artin algebras $\Lambda$ ?

In this paper, we show that this question has a positive answer when $\Lambda$ has radical square zero. Our main result is:

Theorem (Thm. 4.3). Let $\Lambda$ be an Artin algebra with radical square zero. Then

$$
\text { Findim } \Lambda^{o p}=\operatorname{dell} \Lambda \text {. }
$$

We additionally give a combinatorial formula for $\operatorname{dell} \Lambda$, and therefore for Findim $\Lambda^{o p}$, in terms of the valued quiver of $\Lambda$ (Proposition 3.6). This theorem gives some hope that the answer to Question 4.2 should be positive in general.

Conventions and notation. By an Artin algebra $\Lambda$, we will always mean an $R$ algebra $\Lambda$ which is module finite over a commutative Artinian ring $R$. We let $\operatorname{Mod} \Lambda$ stand for the category of right $\Lambda$-modules, and $\bmod \Lambda$ for the full subcategory of finitely presented right modules. We identify left modules with right modules over the opposite ring $\Lambda^{o p}$. Without further description, the term module always refers to a finitely presented right $\Lambda$-module. We let $D: \bmod \Lambda \rightarrow \bmod \Lambda^{o p}$ stand for the duality between right and left $\Lambda$-modules, see [ARS95, II.3].

We denote by $\underline{\bmod } \Lambda$ the stable module category of $\Lambda$, whose objects agree with those of $\bmod \Lambda$ and whose morphisms are module homomorphism modulo homomorphisms factoring through projectives. We refer to the retracts and isomorphisms in $\underline{\bmod } \Lambda$ as stable retracts and stable equivalences, respectively. It is well known that, for $X, Y \in \bmod \Lambda$, the module $X$ is a stable retract of $Y$ in $\underline{\bmod } \Lambda$ if and only if $X$ is 
a retract of $Y \oplus P$ in $\bmod \Lambda$ for some projective $P$, and moreover $X, Y$ are stably equivalent if and only if $X \oplus Q \cong Y \oplus P$ in $\bmod \Lambda$ for some projectives $P, Q$ (see e.g. [Gé20, Lemma 0.1]).

\section{THE MAIN INVARIANTS}

Let $\Lambda$ be an Artin algebra with radical $r$ satisfying $r^{2}=0$. From now on $\Lambda$ will always denote such an algebra with radical square zero, unless specified otherwise. We write $\left\{T_{1}, T_{2}, \ldots, T_{n}\right\}$ for a complete set of simple left modules in $\bmod \Lambda^{o p}$, and similarly $\left\{S_{1}, S_{2}, \ldots, S_{n}\right\}$ for a complete set of simple right modules in $\bmod \Lambda$. The little and big finitistic dimensions of $\Lambda$ are defined respectively by

$$
\begin{aligned}
\operatorname{findim} \Lambda & =\sup \{\operatorname{pdim} M \mid M \in \bmod \Lambda \text { and } \operatorname{pdim} M<\infty\} \\
\text { Findim } \Lambda & =\sup \{\operatorname{pdim} M \mid M \in \operatorname{Mod} \Lambda \text { and } \operatorname{pdim} M<\infty\}
\end{aligned}
$$

More precisely these are the right finitistic dimensions of $\Lambda$, and the left finitistic dimensions are given by findim $\Lambda^{o p}$ and Findim $\Lambda^{o p}$. We will be particularly interested in the latter.

We next define the invariant dell $\Lambda$, called "delooping level" in [Gé20]. The reader is referred to [Gé20, Section 1] for more details. First, we let

$$
\Omega: \underline{\bmod } \Lambda \rightarrow \underline{\bmod } \Lambda
$$

denote the syzygy functor on the stable module category, which can computed on objects as the kernel $\Omega M=\operatorname{ker}(P \rightarrow M)$ of an epimorphism from a projective. Let

$$
\Sigma: \underline{\bmod } \Lambda \rightarrow \underline{\bmod } \Lambda
$$

be the left adjoint to $\Omega$, which was introduced by Auslander-Reiten [AR96]. Since the pair $(\Sigma, \Omega)$ consists of adjoint endofunctors, taking powers gives rise to a series of adjoint pairs $\left(\Sigma^{n}, \Omega^{n}\right)$ for each $n \geq 0$. Each module $M \in \underline{\bmod } \Lambda$ then admits a unit and counit map

$$
\begin{aligned}
& \eta_{n}: M \rightarrow \Omega^{n} \Sigma^{n} M \\
& \varepsilon_{n}: \Sigma^{n} \Omega^{n} M \rightarrow M .
\end{aligned}
$$

To define dell $\Lambda$, we first define an invariant dell $S$ for simple modules $S \in \bmod \Lambda$ taking value in $\mathbb{N} \cup\{\infty\}$.

Definition-Theorem 1.1 ([Gé20, Def. 1.2, Thm. 1.10]). The following are equivalent for dell $S$ :

a) $\operatorname{dell} S \leq n$.

b) $\Omega^{n} S$ is a stable retract of $\Omega^{n+1} N$ for some $N \in \bmod \Lambda$.

c) $\Omega^{n} S$ is a stable retract of $\Omega^{n+1} \sum^{n+1} \Omega^{n} S$.

d) The unit map $\eta_{n+1}: \Omega^{n} S \rightarrow \Omega^{n+1} \Sigma^{n+1} \Omega^{n} S$ is a split-monomorphism.

Remark 1.2. In this article we shall focus on condition b) as a mean of computing dell $S$. However, the conditions c)-d) are of great practical and theoretical interest in general, and so we include them as part of the definition.

We then define dell $\Lambda$ by taking supremum over the simples $S$ in $\bmod \Lambda$.

Definition 1.3. The delooping level of $\Lambda$ is defined as dell $\Lambda:=\sup _{S} \operatorname{dell} S$. 
Unpacking the definition, we see that dell $\Lambda \leq n$ if and only if $\Omega^{n} S$ is a stable retract of $\Omega^{n+1} N$ for some $N \in \bmod \Lambda$ for each simple $S \in \bmod \Lambda$.

Finally, we note that Findim $\Lambda^{o p} \leq \operatorname{dell} \Lambda$ for any Artin algebra ([Gé20, Prop. 1.3]). To investigate whether this is an equivalence in our setting, we first find methods to calculate each side.

\section{ThE FINITISTIC DIMENSION OF RADICAL SQUARE ZERO ALGEBRAS}

We can compute the finitistic dimensions of $\Lambda^{o p}$ in terms of the projective dimensions of left simple modules. Define the invariant $s$ as

$$
s=\sup \left\{\operatorname{pdim} T \mid T \in \bmod \Lambda^{o p} \text { simple with } \operatorname{pdim} T<\infty\right\} .
$$

Mochizuki [Moc65] showed the following (see also [HZ95, Section 3]).

Proposition 2.1 ([Moc65]). We have (in)equalities

$$
s \leq \text { findim } \Lambda^{o p}=\text { Findim } \Lambda^{o p} \leq s+1 .
$$

Since the little and big finitistic dimensions coincide in our situation, in the sequel we only focus on Findim $\Lambda^{o p}$. We can decide whether Findim $\Lambda^{o p}=s$ or Findim $\Lambda^{o p}=s+1$ as follows.

Lemma 2.2. The following are equivalent:

i) Findim $\Lambda^{o p}=s$.

ii) Every left simple $T$ with $\operatorname{pdim} T=s$ satisfies $T^{*}=0$.

Proof. $i) \Longrightarrow i i$ ). Let $T$ be a simple module with $\operatorname{pdim} T=s$. If $T^{*} \neq 0$, then there exists a non-zero morphism $\varphi: T \rightarrow{ }_{\Lambda} \Lambda$ which is then an embedding as $T$ is simple. Letting $\operatorname{coker}(\varphi)=N$ gives $\Omega N=T$ and $\operatorname{pdim} N=s+1$, contradicting i).

ii $\Longrightarrow i$ ). Assume that Findim $\Lambda^{o p} \neq s$, so that Findim $\Lambda^{o p}=s+1$. Let $M$ be a left module with pdim $M=s+1$. Let $\pi: P \rightarrow M$ be its projective cover. Then $\Omega M=\operatorname{ker}(\pi) \subseteq \mathrm{r} P$ is annihilated by $\mathrm{r}\left(\right.$ as $\left.\mathrm{r}^{2}=0\right)$ and so is semisimple. Since $\operatorname{pdim} \Omega M=s$, there exists a simple summand $T$ of $\Omega M$ with $\operatorname{pdim} T=s$. But then $T \subseteq P$ implies $T^{*} \neq 0$, contradicting ii).

\section{The DELOOPING LEVEL OF RADICAL SQUARE ZERO ALGEBRAS}

Next, we consider the computation of dell $\Lambda$; we will obtain a combinatorial formula for this invariant in terms of the valued quiver of $\Lambda$. We will then be able to match the combinatorial formula to the description of Findim $\Lambda^{o p}$ above.

Recall from [ARS95, III.1] that the valued quiver $\Gamma=\left(\Gamma_{0}, \Gamma_{1}\right)$ of $\Lambda$ consists of a set of vertices $\Gamma_{0}=\{1,2, \ldots, n\}$, and $\Gamma_{1}$ consists of a set of arrows between vertices $(i, j)$, precisely one arrow $\alpha: j \rightarrow i$ for each pair such that $\operatorname{Ext}_{\Lambda}^{1}\left(S_{i}, S_{j}\right) \neq 0$. The endomorphism ring of each simple $\Delta_{i}=\operatorname{End}_{\Lambda}\left(S_{i}\right)$ is a division algebra, which we use to equip each arrow $j \rightarrow i$ with a valuation, namely a pair of integers $\left(m_{i}, m_{j}\right)$ defined as

$$
\begin{aligned}
& m_{i}=\operatorname{dim}_{\Delta_{i}} \operatorname{Ext}_{\Lambda}^{1}\left(S_{i}, S_{j}\right) \\
& m_{j}=\operatorname{dim}_{\Delta_{j}} \operatorname{Ext}_{\Lambda}^{1}\left(S_{i}, S_{j}\right) .
\end{aligned}
$$


Note that using the duality $D: \bmod \Lambda \rightarrow \bmod \Lambda^{o p}$, one sees that the valued quiver of $\Lambda^{o p}$ is the opposite quiver $\Gamma^{o p}$ obtained by reversing arrows and valuations $\left(m_{i}, m_{j}\right) \leftrightarrow\left(m_{j}, m_{i}\right)$. When $\Lambda=k Q / \mathrm{r}^{2}$ is a quiver path algebra over a field $k$, the valued quiver $\Gamma$ has the same vertices as $Q$ and has, for every pair of vertices $(i, j)$ with $m$ arrows $j \rightarrow i$, a single arrow with valuation $(m, m)$.

In general we can use the valuation quiver to encode the resolutions of simple modules.

Proposition 3.1 ([ARS95, Prop. 1.15 a)]). Let $\Lambda$ be an Artin algebra with radical r. Let $P_{i} \rightarrow S_{i}$ and $P_{j} \rightarrow S_{j}$ be the projective covers of the simple modules $S_{i}$ and $S_{j}$ respectively. Then the following numbers are the same.

i) $m_{j}=\operatorname{dim}_{\Delta_{j}} \operatorname{Ext}_{\Lambda}^{1}\left(S_{i}, S_{j}\right)$.

ii) The multiplicity of the simple module $S_{j}$ as a summand of $P_{i} \mathrm{r} / P_{i} \mathrm{r}^{2}$.

Since in our situation $r^{2}=0$, this provides a description of the syzygies of simple modules.

Corollary 3.2. For each simple $S_{i} \in \bmod \Lambda$ we have a short exact sequence

$$
0 \rightarrow \bigoplus_{j \rightarrow i} S_{j}^{m_{j}} \rightarrow P_{i} \rightarrow S_{i} \rightarrow 0
$$

where the sum is over all arrows $j \rightarrow i$ in $\Gamma$.

Proof. We have a short exact sequence

$$
0 \rightarrow P_{i} \mathrm{r} \rightarrow P_{i} \rightarrow S_{i} \rightarrow 0
$$

and $P_{i} \mathrm{r}=\bigoplus_{j \rightarrow i} S_{j}^{m_{j}}$ by the last proposition since $\mathrm{r}^{2}=0$.

We now have a description of the first syzygies of $S_{i}$ as

$$
\Omega S_{i}=\bigoplus_{j \rightarrow i} S_{j}^{m_{j}}
$$

Iterating Corollary 3.2, we obtain a description of the higher syzygies of simple modules.

Corollary 3.3. The higher syzygies of simples $S_{i} \in \bmod \Lambda$ are given by

$$
\Omega^{n} S_{i}=\bigoplus_{j \rightarrow j_{1} \rightarrow \cdots \rightarrow j_{n-1} \rightarrow i} S_{j}^{m}
$$

where the sum is over all paths of length $n$ from $j$ to $i$ in $\Gamma$ and the multiplicity is $m:=m_{j} m_{j_{1}} \ldots m_{j_{n-1}}$.

We next aim to describe the delooping level of the simples $S_{i}$. Recall that this is given by

$$
\text { dell } S_{i}=\inf \left\{n \geq 0 \mid \Omega^{n} S_{i} \text { is a stable retract of } \Omega^{n+1} N \text { for some } N \in \bmod \Lambda\right\} .
$$

We have that dell $S_{i}=0$ if and only if $S_{i}$ is a retract of a syzygy module, if and only if $S_{i}$ embeds in a projective. Since $S_{i}$ is simple, this is equivalent to $S_{i}^{*} \neq 0$. We can give a combinatorial characterisation of this last condition. Recall that a vertex $i \in \Gamma_{0}$ is a source if there are no arrows $j \rightarrow i$, and is a sink if there are no arrows $i \rightarrow j$. 
Lemma 3.4. Let $i \in \Gamma_{0}$ be a vertex.

a) $i$ is a sink in $\Gamma$ if and only if $S_{i}$ is injective.

b) $i$ is a source in $\Gamma$ if and only if $S_{i}$ is projective.

Proof. By definition $i$ is a sink if and only if there are no arrows $i \rightarrow j$ in $\Gamma$, if and only if $\operatorname{Ext}_{\Lambda}^{1}\left(S_{j}, S_{i}\right)=0$ for all simples $S_{j}$, if and only if $S_{i}$ is injective. This proves a), and the proof of b) is dual.

Lemma 3.5. The following are equivalent for a vertex $i \in \Gamma_{0}$ :

a) dell $S_{i}=0$.

b) $S_{i}^{*} \neq 0$.

c) $i$ is a source or not a sink.

Proof. We saw the equivalence $a) \Longleftrightarrow b$ ) in the paragraph above Lemma 3.4.

b) $\Longrightarrow c)$ : Let $\varphi: S_{i} \rightarrow \Lambda_{\Lambda}$ be a non-zero morphism. Since $S_{i}$ is simple, $\varphi$ is an embedding of $S_{i}$ into $\Lambda_{\Lambda}$. Now assume that $i$ is a sink. By the Lemma 3.4, the simple $S_{i}$ is injective, and therefore the short exact sequence

$$
0 \rightarrow S_{i} \stackrel{\varphi}{\rightarrow} \Lambda_{\Lambda} \rightarrow \operatorname{coker} \varphi \rightarrow 0
$$

splits. This shows that $S_{i}$ is projective and therefore $i$ is a source by Lemma 3.4. Hence we have shown that if $i$ is a sink, then it must be a source, and c) holds.

$c) \Longrightarrow b)$. If $i$ is a source then $S_{i}$ is projective and so $S_{i}^{*} \neq 0$. If $i$ is not a sink, then there is an arrow $i \rightarrow j$ in $\Gamma$ and therefore $\operatorname{Ext}_{\Lambda}^{1}\left(S_{j}, S_{i}\right) \neq 0$. By Corollary 3.2, the simple $S_{i}$ is a summand of $\Omega S_{j}$. Since $\Omega S_{j}$ embeds in a projective, so does $S_{i}$ and thus $S_{i}^{*} \neq 0$. Hence in either situation b) holds.

For the higher case, we have the next proposition.

Proposition 3.6. The following are equivalent for the vertex $i \in \Gamma_{0}$ :

a) dell $S_{i} \leq n$.

b) For every path of length $n$

$$
j \rightarrow j_{1} \rightarrow \cdots \rightarrow j_{n-1} \rightarrow i
$$

either $j$ is a source, or there exists a path of length $n$

$$
j \rightarrow j_{1}^{\prime} \rightarrow \cdots \rightarrow j_{n-1}^{\prime} \rightarrow k
$$

with $k$ not a sink.

Proof. Lemma 3.5 already covers the case $n=0$ and so we may assume that $n \geq 1$.

a) $\Longrightarrow b)$. Assuming dell $S_{i} \leq n$, then $\Omega^{n} S_{i}$ is a stable retract of $\Omega^{n+1} N$ for some $N \in \bmod \Lambda$. Since $r^{2}=0$, the first syzygy $\Omega N$ is semisimple, say with decomposition $\Omega N=\bigoplus_{k} S_{k}$. Each such simple $S_{k}$ then satisfies $S_{k}^{*} \neq 0$, and so $k$ is either a source (if $S_{k}$ is projective) or not a sink. We then have

$$
\Omega^{n+1} N=\bigoplus_{k} \Omega^{n} S_{k} .
$$


Moreover as $n \geq 1$, we may ignore those $k$ in the decomposition above with $S_{k}$ projective and therefore assume that for each $S_{k}$, the vertex $k$ is not a sink. Expanding this out using Corollary 3.3, we obtain a decomposition

$$
\Omega^{n+1} N=\bigoplus_{k} \bigoplus_{j^{\prime} \rightarrow j_{1}^{\prime} \rightarrow \cdots \rightarrow j_{n-1}^{\prime} \rightarrow k} S_{j^{\prime}}^{m^{\prime}}
$$

running over all paths of length $n$ in $\Gamma$, and with $m^{\prime}$ the multiplicity. Similarly we have

$$
\Omega^{n} S_{i}=\bigoplus_{j \rightarrow j_{1} \rightarrow \cdots \rightarrow j_{n-1} \rightarrow i} S_{j}^{m} .
$$

Since $\Omega^{n} S_{i}$ is a stable retract of $\Omega^{n+1} N$, there exists a projective $P$ such that $\Omega^{n} S_{i}$ is a module retract of $P \oplus \Omega^{n+1} N$. By the Krull-Schmidt theorem, it follows that every summand $S_{j}$ of $\Omega^{n} S_{i}$ is either projective, in which case $j$ is a source, or occurs as $S_{j}=S_{j^{\prime}}$ in the decomposition of $\Omega^{n+1} N$ above, in which case there is a path

$$
j=j^{\prime} \rightarrow j_{1}^{\prime} \rightarrow \cdots \rightarrow j_{n-1}^{\prime} \rightarrow k
$$

with $k$ not a sink. Hence condition $\mathrm{b}$ ) holds.

$b) \Longrightarrow a$ ). We run the argument in reverse. For each simple summand $S_{j}$ of $\Omega^{n} S_{i}$, we see that either $j$ is a source, or there is a path of length $n$ in $\Gamma$

$$
j \rightarrow j_{1}^{\prime} \rightarrow \cdots \rightarrow j_{n-1}^{\prime} \rightarrow k
$$

with $k$ not a sink. In the first case $S_{j}$ is projective, and in the second case $S_{j}$ is a summand of $\Omega^{n} S_{k}$ with $S_{k}$ a simple satisfying $S_{k}^{*} \neq 0$. But then there is an embedding $\varphi_{k}: S_{k} \hookrightarrow \Lambda_{\Lambda}$, and letting $N_{k}=\operatorname{coker} \varphi_{k}$, we obtain $S_{k}=\Omega N_{k}$.

Letting $P=\bigoplus_{j} S_{j}$ be the sum of those $S_{j}$ which were projective (in the first case) and $N=\bigoplus_{k} N_{k}$ (from the second case), we obtain that $\Omega^{n} S_{i}$ is a retract of $P \oplus \Omega^{n+1} N$. This proves that dell $S_{i} \leq n$ and condition a) holds.

The previous proposition gives a combinatorial formula for dell $S_{i}$ for each simple $S_{i}$, and therefore also a formula for dell $\Lambda=\sup _{i} \operatorname{dell} S_{i}$.

\section{Proof of the MAIN THEOREM}

To compare dell $\Lambda$ to Findim $\Lambda^{o p}$, recall that we have defined the invariant

$$
s=\sup \left\{\operatorname{pdim} T \mid T \in \bmod \Lambda^{o p} \text { simple with } \operatorname{pdim} T<\infty\right\}
$$

and that we always have $s \leq$ Findim $\Lambda^{o p} \leq s+1$. We can recast the value $s$ combinatorially in terms of the valued quiver of $\Lambda$ as follows. First, given a vertex $j \in \Gamma_{0}$, denote by $\downarrow j \subseteq \Gamma$ the full subquiver consisting of vertices $i$ reachable by a path $j \rightarrow \cdots \rightarrow i$. When $\downarrow j \subseteq \Gamma$ is a subquiver without oriented cycle, we denote by $\ell(\downarrow j)$ the length of the longest path in it starting from $j$.

Lemma 4.1. We have $s=\sup \{\ell(\downarrow j) \mid$ the subquiver $\downarrow j \subseteq \Gamma$ has no oriented cycle $\}$.

Proof. To each vertex $j \in \Gamma_{0}$ we let $T_{j} \in \bmod \Lambda^{o p}$ be the associated left simple module. Applying Corollary 3.3 to the simple $T_{j}$ we see that simple summands of 
$n$-th syzygies of $T_{j}$ are given by

$$
\Omega^{n} T_{j}=\bigoplus_{i \rightarrow i_{1} \rightarrow \cdots \rightarrow i_{n-1} \rightarrow j} T_{i}^{m} .
$$

Hence the simples $T_{i}$ occuring as summands of syzygies of $T_{j}$ are those whose vertex $i$ can reach $j$ by a path in the valuation quiver $\Gamma^{o p}$ of $\Lambda^{o p}$. Since the latter reverses orientations, these are precisely the vertices $i \in \downarrow j \subseteq \Gamma$.

The above description of the syzygies then shows that $\operatorname{pdim} T_{j}<\infty$ if and only $\downarrow j$ has no oriented cycle, in which case $\operatorname{pdim} T_{j}=\ell(\downarrow j)$. The formula then holds.

We can now refine the inequality $s \leq$ Findim $\Lambda^{o p} \leq s+1$.

Proposition 4.2. We have $s \leq$ Findim $\Lambda^{o p} \leq \operatorname{dell} \Lambda \leq s+1$.

Proof. We always have Findim $\Lambda^{o p} \leq \operatorname{dell} \Lambda$ and so it suffices to show dell $\Lambda \leq s+1$.

By contradiction, assume that dell $\Lambda>s+1$, so that dell $S_{i}>s+1$ for some right simple $S_{i}$. By Proposition 3.6, we conclude that there exists a path of length $s+1$ in $\Gamma$

$$
j \rightarrow j_{1} \rightarrow \cdots \rightarrow j_{s-1} \rightarrow j_{s} \rightarrow i
$$

such that $j$ is not a source, and such that every path of length $s+1$

$$
j \rightarrow j_{1}^{\prime} \rightarrow \cdots \rightarrow j_{s-1}^{\prime} \rightarrow j_{s}^{\prime} \rightarrow k
$$

ends at a sink vertex $k$.

By the second condition, we conclude that the subquiver $\downarrow j \subseteq \Gamma$ has no oriented cycle since it contains no path of length $\geq s+2$. Since there is a path of length $s+1$ from $j$ to $i$, we conclude that $\ell(\downarrow j)=s+1$. But this contradicts maximality of $s$ in Lemma 4.1.

Finally, we arrive at the proof of the main theorem.

Theorem 4.3. Let $\Lambda$ be an Artin algebra with radical square zero. Then we have

$$
\text { Findim } \Lambda^{o p}=\operatorname{dell} \Lambda \text {. }
$$

Proof. If dell $\Lambda=s$ the inequality $s \leq$ Findim $\Lambda^{o p} \leq \operatorname{dell} \Lambda \leq s+1$ gives the result, and so we may assume that dell $\Lambda=s+1$. We will show that Findim $\Lambda^{o p}=s+1$ using a similar argument to the previous proof.

Since dell $\Lambda>s$, by Proposition 3.6 we see that there exists a sink $i \in \Gamma_{0}$ and a path of length $s$ in $\Gamma$

$$
j \rightarrow j_{1} \rightarrow \cdots \rightarrow j_{s-1} \rightarrow i
$$

such that $j$ is not a source in $\Gamma$, and for which every path of length $s$

$$
j \rightarrow j_{1}^{\prime} \rightarrow \cdots \rightarrow j_{s-1}^{\prime} \rightarrow k
$$

ends in a sink vertex $k$. As seen above, this implies that $\operatorname{pdim} T_{j}=\ell(\downarrow j)=s$.

We now claim that $T_{j}^{*} \neq 0$. Note that the condition that $j$ is not a source in $\Gamma$ means that $j$ is not a sink in $\Gamma^{o p}$. Applying Lemma 3.5 to $\Lambda^{o p}$, we obtain that $T_{j}^{*} \neq 0$ as claimed. Finally, since $T_{j}$ is simple there is an embedding $\varphi: T_{j} \hookrightarrow \Lambda^{o p}$, 
THE FINITISTIC DIMENSION FOR ALGEBRAS WITH RADICAL SQUARE ZERO

and letting $N=\operatorname{coker}(\varphi)$, we obtain $\Omega N=T_{j}$ and so that $\operatorname{pdim} N=s+1$. This shows that $s+1 \leq$ Findim $\Lambda^{o p} \leq \operatorname{dell} \Lambda=s+1$, and so Findim $\Lambda^{o p}=\operatorname{dell} \Lambda$.

\section{REFERENCES}

[AHHT06] Lidia Angeleri-Hügel, Dolors Herbera, and Jan Trlifaj. Tilting modules and Gorenstein rings. Forum Math., 18(2):211-229, 2006.

[AR96] Maurice Auslander and Idun Reiten. Syzygy Modules for Noetherian Rings. Journal of Algebra, 183(1):167 - 185, 1996.

[ARS95] Maurice Auslander, Idun Reiten, and Sverre O. Smalo. Representation Theory of Artin Algebras. Cambridge Studies in Advanced Mathematics. Cambridge University Press, 1995.

[Bas62] Hyman Bass. Injective Dimension in Noetherian Rings. Transactions of the American Mathematical Society, 102(1):18-29, 1962.

[GR71] L. Gruson and M. Raynaud. Critères de platitude et de projectivité: Techniques de "platification" d'un module. Inventiones mathematicae, 13:1-89, 1971.

[Gé20] V. Gélinas. The depth, the delooping level and the finitistic dimension. arxiv:2004.04828, 2020.

[HZ95] B. Huisgen-Zimmermann. The finitistic dimension conjectures - a tale of 3.5 decades. Abelian Groups and Modules (A. Facchini and C. Menini, Eds.), pages 501-517, 1995.

[Jan61] J. P. Jans. Some generalizations of finite projective dimension. Illinois J. Math., 5(2):334-344, 061961.

[Moc65] Horace Mochizuki. Finitistic global dimension for rings. Pacific J. Math., 15(1):249$258,1965$.

VINCENT.GELINAS@MAIL.UTORONTO.CA 